\title{
Modelo de argumentación técnica para el dictamen de patología de la edificación
}

\section{Technical argument in the building technical pathology report}

\author{
$\underline{\text { L. Gómez-Martinho Palacio }}^{(*)}$, F. Lasheras Merino ${ }^{(*)}$
}

\section{RESUMEN}

En el campo de la construcción, un dictamen de patología es un documento en el que un técnico experto en esta disciplina traslada su opinión técnica a un profano o a otro menos experto que él. Para que el destinatario confíe en la opinión del técnico, ha de apreciar coherencia interna y verosimilitud en el dictamen, entre otras cosas. Los conocimientos científicos y técnicos que se le presuponen al autor del dictamen no son suficientes, porque la mejor argumentación técnica puede no transmitir adecuadamente su contenido. Así, es posible y deseable utilizar criterios lógicos de argumentación complementarios a los puramente técnicos, para potenciar la transparencia y comprensibilidad del dictamen, para lo que presentamos un modelo de argumentación técnica desarrollado a partir de otros modelos de argumentación lógica, especialmente del expuesto por Stephen Toulmin en 1979, que se ha aplicado a diferentes campos, pero no, que sepamos, al de patología en edificación.

Palabras clave: Argumentación, dictamen, informe técnico, lógica, patología de la edificación, peritación, prueba.

\section{ABSTRACT}

In the field of construction, a technical pathology report is a document where a technical expert in this discipline makes known their technical opinion thereon to a layman who has no real understanding or to another expert with less knowledge. In order for the addressee to trust the technician's opinion, it's necessary to appreciate internal consistency and plausibility in the report, among other things. Scientific and technical knowledge, presupposed in the author is not enough because the best technical argumentation can not properly transmit its content. Therefore, it's possible and necessary to introduce argument criteria, supplementary to purely technical ones, in order to improve transparency and comprehensibility in the report. For this reason, we are presenting a technical argumentation model, developed from others logic argument models, especially Toulmin's one. It was exposed in 1979 and has been applied in very different fields, but not, unless we know, in building pathology.

Keywords: Argument, expert opinion, technical report, logic, building pathology, evidence.

(*) Escuela Técnica Superior de Arquitectura. Universidad Politécnica de Madrid (UPM) (España).

Persona de contacto/Corresponding author: l.gmartinho.p@gmail.com (L. Gómez-Martinho Palacio).

ORCID: http://orcid.org/oooo-0oo3-3009-5824 (L. Gómez-Martinho Palacio); http://orcid.org/oooo-0002-1252-5360 (F. Lasheras Merino)

Cómo citar este artículo/Citation: Gómez-Martinho Palacio, L.; Lasheras Merino, F. (2020). Modelo de argumentación técnica para el dictamen de patología de la edificación. Informes de la Construcción, 72(557): e327. https://doi.org/10.3989/ic.68833

Copyright: (c) 2020 CSIC. Este es un artículo de acceso abierto distribuido bajo los términos de la licencia de uso y distribución Creative Commons Reconocimiento 4.0 Internacional (CC BY 4.0). 


\section{INTRODUCCIÓN. JUSTIFICACIÓN DE LA INVESTIGACIÓN}

La argumentación en el dictamen técnico se enfoca directamente a la prueba. No sólo él mismo puede ser considerado como prueba en un ámbito jurídico, sino que toda su redacción está volcada a probar la veracidad de sus afirmaciones y la universalidad de sus opiniones. El técnico describe unos hechos (daños materiales o pérdida de prestaciones técnicas del edificio, y defectos constructivos) y opina sobre ellos (relevancia técnica del problema, causa y origen, y posibilidades de reparación), pero, además, tiene que dar prueba suficiente, y convincente, de la realidad de los hechos y de la corrección de sus opiniones técnicas.

En ocasiones, los elementos de prueba que se aportan para justificar la opinión emitida son excesiva o exclusivamente técnicos. Por su propia naturaleza, éstos pueden ser difícilmente comprensibles por el destinatario del informe y, en consecuencia, ser insuficientes para convencerle y garantizarle objetivamente la corrección de la opinión manifestada por el técnico. Ante la incomprensión, se ignora su opinión o, peor aún, se duda de su credibilidad bajo el pensamiento de que la oscuridad oculta la falsedad. Para alguien que no entienda muy bien las implicaciones de los argumentos técnicos de un dictamen, la credibilidad de este no reside en los criterios técnicos expuestos, sino en el hecho de que sus bases sean suficientemente explícitas como para que un tercero que sí disponga de conocimientos técnicos pueda verificarlas.

El destinatario del dictamen suele carecer de conocimientos técnicos suficientes para entender todas las implicaciones y consecuencias de los temas técnicos tratados; en caso contrario no necesitaría el dictamen. Por ello, es necesario dotar de credibilidad al dictamen a partir de criterios diferentes a los meramente técnicos. La forma y la estructura lógica del dictamen son aspectos muy importantes para dar a sus argumentaciones la apariencia de ser completas. Disponer de un esquema lógico del proceso de argumentación técnica es una gran ayuda para quien se dispone a criticar o a redactar un dictamen; le marca un camino a seguir en la exposición de hechos y opiniones con la confianza de no olvidar ningún elemento esencial para dotar a su argumentación de la corrección lógica requerida, sin dejar cabos sueltos y, además, le permite analizar informes contradictorios, comprobar su coherencia y lógica de razonamiento, detectar los elementos sensibles de ser contra-argumentados y, a la vez, marcarle las estrategias a seguir para plantear la argumentación contraria.

Esta investigación surge de considerar que un dictamen técnico no pertenece sólo al campo técnico especializado sobre el que se dictamina, sino también al ámbito argumental y más, concretamente, a sus planos lógico, dialéctico y retórico. La integración de estos tres planos en el dictamen pericial de patología de la edificación ya la presentamos en otro foro (1), aunque pretendemos completarla en futuras publicaciones. En esta, nos centraremos únicamente en el campo lógicoargumental, que da soporte a la información y opiniones que se pretenden transmitir.

El dictamen tiene que ser tan técnicamente riguroso como fácilmente comprensible. Se trata de un tema en la intersección entre dos campos aparentemente muy distintos, y es precisamente en esta zona común en la que muchas veces aparecen conflictos no resueltos, lo que deriva en la incomprensión del dictamen técnico por parte de un destinatario que carece de conocimientos técnicos, pero no de pensamiento lógico.

En el dictamen técnico es muy importante la forma de comunicar. Autores muy reconocidos en el campo de la patología de la edificación han advertido el tema. Por citar tan sólo tres ejemplos, Álvaro García Meseguer (2)(3) trató el problema en publicaciones como Léxico de la Construcción y La patología y el lenguaje, Informes de patología ; José Calavera (4) advirtió que la comunicación es una actividad esencial en el mundo actual y en especial en el complejo mundo de la construcción; y autores de otros campos, como el jurista José Guerra San Martín (5), planteó la dicotomía existente entre la ciencia jurídica y la técnica y la necesidad de acercarlas en beneficio mutuo.

Existen también tesis doctorales en el ámbito técnico y en el campo del derecho que han tratado diferentes aspectos del dictamen pericial en el ámbito jurídico. Destacamos las tesis de David Marín García (6) que se centra en el lenguaje gráfico utilizado en el campo de la peritación judicial en patología en edificación, la de Iñigo León Cascante (7) que trata sobre la figura del perito y su intervención en el proceso judicial, y las de Manuel Matos de Araújo Chaves (8) y Carmen Vázquez Rojas (9), ambas sobre la prueba pericial y la motivación judicial desde la perspectiva del derecho.

Sin embargo, ninguno de los autores precedentes ha analizado la lógica de la argumentación técnica en la práctica profesional y, hoy, el tema sigue abierto. Es indudable que compete al técnico demostrar la racionalidad de su investigación, justificar la veracidad de sus conclusiones, y convencer de sus afirmaciones técnicas a los destinatarios de su dictamen y a sus colegas.

\section{OBJETIVO DE TRABAJO}

Identificamos en este trabajo los elementos principales de los argumentos técnicos para el dictamen en patología de la edificación, analizamos sus relaciones, y desarrollamos una herramienta gráfica que representa argumentos y relaciones para ayudar a desarrollar los argumentos propios y a analizar los ajenos.

Los temas esenciales en estos dictámenes son los siguientes:

- 1) Identificar y describir los daños materiales y los fallos prestacionales asociados, en su realidad fáctica y en su evolución temporal. Ambos aspectos determinan la problemática patológica del caso, especialmente en lo que respecta a los fallos, es decir al incumplimiento de las prestaciones técnicas requeridas al edificio.

- 2) Determinar las causas (acciones incidentes y/o defectos constructivos) y sus orígenes (errores de los agentes de la edificación). Estos dos aspectos determinan las estrategias de reparación, que tendrán que subsanar la causa y reparar los daños.

- 3) Indicar las opciones y el coste de la posible reparación. Ambos temas determinan las posibilidades de solucionar el problema patológico y, en la práctica, son los que más interesan al destinatario del dictamen.

Concretar y explicar estas materias suele requerir una gran especialización en el campo específicamente técnico y en el lógico-argumental, para lo que desarrollaremos y aplicaremos un modelo de argumentación lógico-técnica que nos guíe en estos tres temas duales. 


\section{METODOLOGÍA}

\subsection{Desarrollo del modelo de argumento de S. Toulmin}

La lógica de la argumentación es un tema extensamente tratado desde los primeros filósofos griegos. En nuestro caso, y para el nivel lógico-argumental de un dictamen técnico, partimos de la conocida metodología de argumentación desarrollada por Stephen Toulmin ${ }^{1}$ a mediados del siglo pasado (10) (11), que distingue en una argumentación cuatro proposiciones principales y dos complementarias, que en su forma más simple pueden ser otros tantos enunciados que atribuyen un predicado a un sujeto, y que para nuestro fin definimos del siguiente modo $^{2}$ :

\section{Enunciados elementales:}

- Pretensión (claim): enunciado expresivo de lo que se pretende convencer o demostrar. Se refiere al sujeto y al predicado principales de la argumentación.

- Soporte (ground): datos o hechos específicos que sostienen a la pretensión en cada caso concreto.

- Garantía (warrants): regla o conjunto de ellas que justifican la pertinencia de los datos como prueba de la pretensión. Pertenece al campo técnico, y puede quedar implícita si el contexto lo permite.

- Fundamento (backing): es la razón que demuestra la validez de la garantía para justificar la pertinencia de los datos y para probar la pretensión. También puede quedar implícita en el contexto.

Enunciados complementarios o modificadores (afectan principalmente a la pretensión):

- Modulador de grado, adjetivador o marcador (qualifier) de la pretensión, generalmente en términos probabilísticos. Suele exponerse al principio de la pretensión, por ejemplo: con total seguridad, la causa de los daños es...

- Las cautelas o reservas (rebuttals and exceptions) que plantean posibles excepciones a la pretensión. Normalmente se exponen al final de ella, por ejemplo: la causa de los daños es..., salvo que tras abrir las calas propuestas se observe que...

Como ambos modificadores afectan a la pretensión, por no complicar el modelo que exponemos, consideraremos que son parte de esta y no los citaremos más.

El modelo de Toulmin ha sido muy bien valorado en muchos campos desde su publicación, y tiene muchos seguidores, pero no se ha aplicado al dictamen técnico ni a una argumentación completa en un caso real, que sepamos. Es un posible modelo para seguir, pero no puede aplicarse extensiva ni intensivamente al discurso habitual, que resultaría extremadamente rígido. Sin embargo, el modelo que proponemos, y que utilizamos, puede salvar ambos aspectos.
En nuestro caso, teniendo en cuenta los temas esenciales del dictamen, las pretensiones y sus sujetos y predicados son, básicamente, los siguientes:

- 1a. Hay deterioros (sujeto) en el edificio con entidad suficiente para calificarlos como daños materiales (predicado).

- 1b. Los fallos o deterioros prestacionales observados (sujeto), o asociados a los daños, afectan (predicado) a las prestaciones técnicas del edificio o de sus instalaciones.

- 2a. Las acciones incidentes y las disposiciones constructivas (sujetos) ${ }^{3}$ del edificio son excesivas o defectuosas, respectivamente, y causa de los daños y fallos descritos (predicado).

- 2b. Los defectos o excesos (sujetos) identificados tienen su origen en defectos de proyecto, de ejecución, o de conservación y uso (predicado).

- 3a. La reparación que se propone (sujeto) es la más adecuada para subsanar los daños (predicado).

- 3b. Dicha reparación (sujeto) costaría el importe indicado (predicado).

El soporte es la prueba material u "objetiva" de la pretensión ${ }^{4}$. También es un predicado que se afirma de un sujeto, y contiene los datos o parámetros técnicos que describen o cuantifican al sujeto de la pretensión. En nuestro caso, usamos los siguientes 5 :

- 1a. Los datos de los daños son su tipo, ubicación, forma, extensión, intensidad y cronología. Suelen complementarse con dibujos o fotografías.

- 1b. Los datos de los fallos son los resultados de análisis, ensayos, mediciones o pruebas. A veces se omiten por su coste de obtención, aunque en su lugar y al menos, debería describirse cualitativa u ordinalmente con la mayor precisión posible.

- 2a. Los datos de las acciones actuantes y de las disposiciones constructivas son los parámetros técnicos relativos a su constitución y valores dimensionales, y de similar naturaleza a los anteriores.

- 2b. Los datos que explican el valor de las acciones o de las condiciones constructivas alcanzadas son los análogos a los anteriores que corresponden a las especificaciones del proyecto o de la dirección de la obra, o a las ejecutadas o alteradas por su conservación o uso, obtenidas de un análisis documental o de una inspección directa.

- 3a. Los datos de la solución técnica propuesta son similares a los anteriores, pero referidos a la reparación.

- 3b. Los datos del coste de reparación son, principalmente, la definición de unidades y partidas de obra, sus mediciones desglosadas y sus precios unitarios descompuestos.

La garantía es la "prueba técnica" de la pretensión. Explica el significado de los datos en el contexto de ésta, les dota de valor técnico y justifica su pertinencia mediante la referencia a un patrón técnico objetivo de comparación, que da sentido a su valoración.

\footnotetext{
${ }^{1}$ Stephen Toulmin (1922-2009) fue un reputado pensador británico, profesor en varias universidades, destacado por su aplicación al campo del razonamiento sin ser jurista.

2 Entre paréntesis, la designación original de Toulmin.

3 Ocasionalmente, puede incluirse el estado previamente deteriorado (daños causales), pero no cambia la esencia de la pretensión.

4 Se excluye aquí cualquier interpretación o valoración técnica, que se lleva a la garantía.

5 Ahorramos al lector la indicación del sujeto y del predicado, que es elemental, por otro lado.
} 
- 1a. Para los daños, hay un doble patrón de referencia: por un lado, el estado original del edificio, recién terminado y documentado ${ }^{6}$; por otro, el que pudiera establecer la normativa técnica de referencia al respecto.

- 1b. Para los fallos también se utilizan los patrones anteriores, aunque los valores de referencia son los parámetros técnicos relativos a las prestaciones originales y requeridas del edificio.

- 2a. Para los defectos constructivos y para las acciones causales el patrón de referencia es la normativa técnica de aplicación: el CTE, la EHE, RITE, etc., y, en su defecto, la llamada lex artis del sector.

- 2b. Los defectos de proyecto, dirección, ejecución, etc., también se determinan a partir de hechos documentados deducidos del proyecto, actas de obra, libro de órdenes, certificaciones, etc., o de la inspección directa, con referencia a la normativa indicada o a la práctica habitual del sector, aunque aquí se califica el resultado de las actuaciones profesionales diferenciadas de los agentes de la edificación.

- 3a. La reparación propuesta se garantiza con la descripción y dimensionado, calculado si es el caso, de las soluciones constructivas propuestas, igualmente referidas a la normativa técnica de referencia o, en su ausencia, a la experiencia previa demostrada al respecto.

- 3b. La garantía del coste de reparación la proporciona la referencia a bases objetivas de precios, adaptadas a las circunstancias del caso.

El fundamento es el principio científico, técnico o jurídico que avala la pertinencia de la valoración técnica de los datos para la pretensión. Consolida a la pretensión ligando al concepto de su predicado el del predicado de la garantía. A veces, el contexto argumental es tan evidente que el fundamento puede quedar sobreentendido sin mayor justificación. También puede exponerse al principio de la argumentación con validez común para sus argumentos elementales.

- $1 \mathbf{a}$ y $\mathbf{1 b}$. Las pretensiones relativas a los daños y a los fallos, sus soportes y sus garantías, se fundamentan en el marco legislativo y normativo que hace posible calificar una realidad fáctica como anómala o deteriorada. Principalmente son el CTE y demás normativa técnica de aplicación, la bibliografía técnica especializada, la LOE y el Código Civil, aunque en algunos casos puede ser necesario recurrir a los principios tecnológicos en los que se basan algunas técnicas analíticas, procedimientos de ensayo, etc.

- $2 \mathbf{a}$ y $\mathbf{2 b}$. Las pretensiones relativas a causas y orígenes de los daños y fallos se fundamentan en su calificación como incumplimientos de la normativa obligatoria de referencia, en cuanto a la disposición constructiva y condiciones de uso para las primeras, y en cuanto al resultado de las actuaciones profesionales diferenciadas de los agentes intervinientes para los segundos. En general, estas referencias se establecen contractualmente en la LOE, en el CTE y en el resto de normativa técnica de aplicación.
- $3 \mathbf{a}$ y $\mathbf{3 b}$. Las pretensiones relativas a la reparación propuesta tienen sus principios fundamentales en su adecuación al marco de referencia anterior y, en cuanto a su valoración económica, al Anejo I del Real Decreto que aprueba el CTE, referido a la justificación de partidas y conceptos valorados.

\subsection{Representación gráfica de la argumentación}

Toulmin representa los elementos principales en un esquema ramificado como el que se muestra a continuación, en el que, por simplificar, hemos eliminado los modificadores antes aludidos (Figura 1):

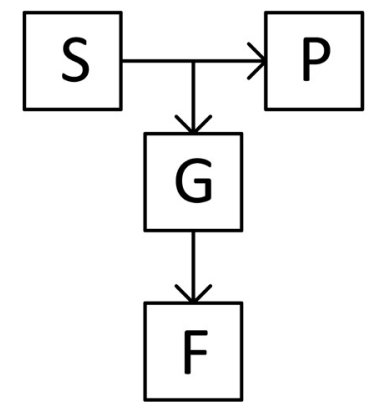

P: pretensión

S: soporte

G: garantía

F: fundamento

Figura 1. Representación gráfica del modelo de Toulmin.

Sin embargo, en este modelo, la representación de una argumentación compleja, con varios argumentos entrelazados, no está fácilmente resuelta.

En nuestro modelo lógico cerramos el argumento para que sea coherente y completo en la medida en que pueda serlo ${ }^{7}$. Esto se consigue enlazando el fundamento con la pretensión de forma que el predicado del fundamento contenga una referencia genérica al predicado de la pretensión, y no particular, para no caer en lo que sería una evidente tautología. Así, planteamos el fundamento en el plano de los principios y la pretensión en el de las realidades fácticas.

Utilizando los cuatro enunciados principales identificados por Toulmin como pasos argumentales, hemos definido diferentes niveles de prueba o soporte para cada uno de ellos, aunque no son objeto de esta publicación.

Otros autores, como Manuel Atienza (12), han tratado de representar gráficamente las argumentaciones, pero se han limitado al ámbito jurídico y no son directamente aplicables a nuestro campo. En el campo técnico, García Meseguer (13) mostró la representación gráfica del árbol de fallos y errores o árbol de defectos ${ }^{8}$ al campo de la patología y del control de calidad.

Toulmin representa las partes del argumento en cajas conectadas con flechas en un esquema lineal ramificado (fig. 1). Sin embargo, en correspondencia con lo que aca-

\footnotetext{
${ }^{6}$ Habitualmente implícito.

7 Una argumentación compleja está formada por sucesivas justificaciones de múltiples pretensiones relacionadas. La pretensión principal se intenta probar con sucesivos enunciados instrumentales que se utilizan como prueba de los anteriores, dando lugar a un proceso que puede ser infinito y que sólo se cierra convencionalmente, cuando el redactor o el destinatario se dan por satisfechos y consideran suficientes los razonamientos o argumentos elementales (pretensiones instrumentales) aportados hasta el momento. Sin embargo, cualquier parte de la argumentación podría ser nuevamente abierta por un tercero que quisiera discutirla con otras razones.

8 Originalmente, sistema de representación de análisis de riesgos concebido y utilizado por primera vez en 1962 por H.A. Watson para evaluar las condiciones de seguridad de los sistemas de tiro de los misiles en el campo de la aviación.
} 
bamos de exponer, nos resulta más útil una representación cerrada de la argumentación, en la que cada argumento elemental corresponda al lado de un cuadrilátero, con su sujeto y su predicado en los vértices, identificados con números, mostrando que el sujeto de un argumento hace referencia al predicado del anterior. Ambos conceptos se representan en cuadrados divididos diagonalmente para indicar si sirven de sujeto (trama de puntos) o de predicado (sin trama) en los argumentos elementales, como indicamos a continuación (Figura 2). Esta distinción es útil en argumentaciones complejas.

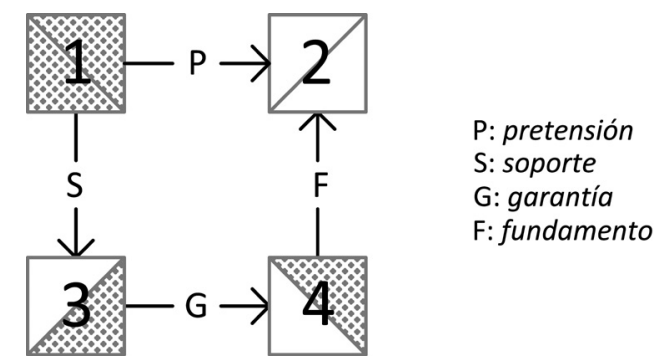

Figura 2. Representación gráfica básica del modelo argumental que proponemos.

Las flechas representan los argumentos elementales: Pretensión (P), Soporte (S), Garantía (G) o Fundamento (F). Las líneas son continuas si un argumento está bien razonado, de trazos en uno implícito aceptable, y la de puntos en los que son ausentes o insuficientes o no pueden ser aceptados (Figura 2).

Queremos destacar que la representación gráfica que proponemos es conceptual, especialmente en sus nodos, cuyo tema de fondo es el que sirve de sujeto o de predicado a cada argumento, que pueden presentar variaciones textuales según en cuál de estos intervengan.

\section{DESARROLLO}

Siguiendo este modelo, y para justificar las pretensiones principales de un dictamen, su redactor desarrollaría la argumentación en varios pasos:

- 1. Pretensión, expresada en la forma "1 es 2 " $(1 \rightarrow 2)$, donde "1" es el sujeto de la pretensión y "2" su predicado con las circunstancias o condiciones que se pretenden defender (vg. el deterioro observado es un daño relevante).

- 2. Soporte (prueba material), reflejando la realidad material de " 1 ", en la forma " 1 es 3 ", siendo " 3 " el conjunto de datos o parámetros técnicos (predicado) que describen " 1 " (sujeto) en relación con "2" (vg. tal deterioro afecta a tales elementos, tiene tal forma, extensión e intensidad, y evolución cronológica).

- 3. ${ }^{\circ}$ Garantía (prueba técnica), en el que la valoración técnica de " 3 " (sujeto) se compara con un patrón de referencia objetivo " 4 " (predicado), que a su vez es válido para el predicado "2" de "1" (vg. los datos del daño son anormales y negativos en comparación con tal patrón de referencia técnica).

- 4. ${ }^{\circ}$ Fundamento, mostrando la pertinencia del patrón de comparación "4" (sujeto) respecto al predicado "2" que se defiende de "1". Dependiendo del contexto de la pretensión, este paso puede desarrollarse con referencia al campo científico, al tecnológico o al jurídico (vg. el patrón, que es de aplicación obligatoria, contiene las especificaciones que permiten calificar al daño como tal).

El técnico podría matizar su pretensión con algún modulador de grado o cautela que condicione la pretensión a determinadas circunstancias, si fuera necesario.

Cualquiera de los pasos anteriores, según las circunstancias del destinatario o de la argumentación, puede requerir argumentaciones derivadas de segundo o sucesivos niveles. También puede ser evidente por su contexto y quedar implícito sin formulación expresa, salvo que alguien lo ponga en duda.

La representación gráfica del modelo básico de argumentación que proponemos admite múltiples variaciones en función de la complejidad de la argumentación completa. La pretensión y los argumentos elementales pueden necesitar enunciados o razonamientos complementarios, que pueden relacionarse en serie (si uno de ellos sirve de paso al siguiente) o en paralelo (en el que, a su vez, los enunciados pueden mantener una relación simultánea, si todos ellos son obligatorios, o complementaria, si suponen diferentes alternativas), o en forma más compleja, porque la variedad y sutileza del razonamiento humano puede ser infinita. En cualquier caso, conocido el esquema argumental y los pasos a seguir, es obligación del técnico transformar este modelo en una redacción coherente y de fácil lectura.

Aplicamos a continuación el modelo argumental a ejemplos diversos y sencillos de los temas característicos del dictamen de patología ${ }^{9}$.

\subsection{Argumentar sobre los daños y los fallos}

En edificación, un daño material es una alteración anormal, perjudicial y permanente ${ }^{10}$ de un elemento constructivo, que va acompañado de la reducción de las prestaciones técnicas de éste, es decir, de un fallo. Daño y fallo son los dos aspectos del deterioro patológico: el daño es su forma material y el fallo su forma prestacional. En el contexto de la LOE (14) y del CTE (15), el enfoque prestacional prevalece sobre el prescriptivo, por lo que un daño debe asociarse a la pérdida de prestaciones técnicas asociada antes que a su apariencia formal. Lo relevante del daño no es su aspecto, sino la reducción de las prestaciones técnicas del elemento afectado: no importa la grieta, sino la pérdida de estabilidad o de estanqueidad, si las hay. En los daños, hay que probar el fallo antes que la materialidad del daño, porque sin el primero el segundo no es patológicamente relevante. A veces, el fallo asociado al daño es tan evidente que basta describir éste para sobreentender el fallo, pero no siempre es así.

En el modelo argumental que proponemos, caben ambos aspectos. Un ejemplo sencillo (y simplificado) respecto a fisuras de retracción del hormigón en un pavimento de un garaje es el siguiente ${ }^{11}$ (Figura 3 y 4):

\footnotetext{
9 Hemos elegido ejemplos heterogéneos que mostraran la variedad posible de aplicaciones.

10 Mientras no se aborde su reparación.
} 


\section{Daños}

- $\mathbf{P}_{\mathbf{d}}$ (Pretensión). Las fisuras del pavimento del garaje [1] $]^{12}$ constituyen un daño material relevante [2].

- $\mathbf{S}_{\mathbf{d}}$ (Soporte). Estas fisuras [1] son longitudinales, sin resaltos ni desprendimientos (forma), siguen el eje de los viales o nacen en las esquinas de arquetas o sumideros (ubicación), su abertura máxima es de $1 \mathrm{~mm}$ (intensidad) y afectan a un 4\% de la superficie del garaje (extensión) [3].

- $\mathbf{G}_{\mathbf{d}}$ (Garantía). Se omite. En España no hay ningún patrón de referencia [4] para valorar las fisuras en revestimientos en función de sus parámetros técnicos [3].

- $\mathbf{F}_{\mathbf{d}}$ (Fundamento). Se omite. Al faltar el patrón de referencia [4] en relación con el daño material [2], la pretensión queda sin fundamento.

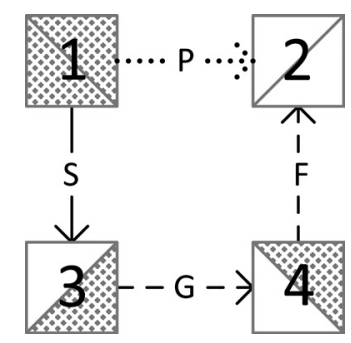

Figura 3. Representación de un argumento incompleto en el que garantía y fundamento se omiten.

\section{Fallos}

- $\mathbf{P}_{\mathbf{f}}$ : La retracción del hormigón [1] no constituye un fallo relevante [2] porque está dentro de los límites admisibles.

- $\mathbf{S}_{\mathbf{f}}$ : La retracción del hormigón [1], a la vista de la amplitud de las fisuras, ha tenido un valor de $1 \mathrm{~mm} / 5 \mathrm{~m}=0,2 \%$ o [3]

- $\mathbf{G}_{\mathbf{f i}}$ : Un valor de 0,2\%o [3] es menor que la retracción normal del hormigón, según art $^{\circ} 39.7$ de la EHE-o8 [4A].

- $\mathbf{G}_{\mathbf{f}_{\mathbf{2}}}$ : Un valor de $0,2 \%$ [3] es menor que la retracción normal del hormigón, según la bibliografía especializada, vg. Jiménez Montoya et. al. [4B].

- $\mathbf{F}_{\mathbf{f i}}$ : La EHE-o8 [4A] es una normativa de obligado cumplimiento que evita, entre otras cosas, la aparición de fallos en la edificación [2].

- $\mathbf{F}_{\mathbf{f}_{2}}$ : El libro de Jiménez Montoya et. al. ${ }^{13}$ [4B] es una referencia científica de reconocido prestigio y contiene criterios para evitar, entre otras cosas, fallos en elementos de hormigón para la edificación [2].

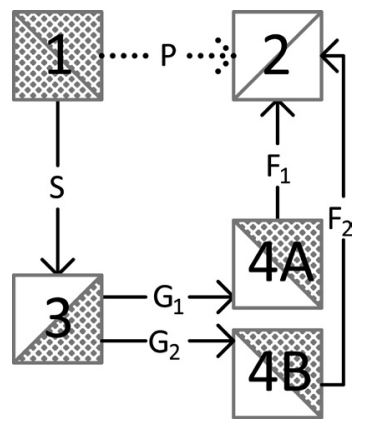

Figura 4. Representación del argumento del fallo del ejemplo.
La Figura 4 muestra cómo la garantía y el fundamento están formados por dobles argumentos complementarios en paralelo, que se refuerzan mutuamente para mejorar su aceptación.

\subsection{Argumentar sobre las causas y sus orígenes}

Apenas hay bibliografía que plantee una metodología general para la determinación de la causa de los daños en patología de la edificación, a pesar de que disponer de una teoría que nos explique la situación a la que nos enfrentamos es el primer paso para poder transformarla intencionalmente, aunque será la práctica la que nos dé el conjunto de conocimientos para poder hacerlo (16).

Varios autores han tratado de ofrecer un marco global de la problemática causal de problemas concretos, pero ninguno aporta una visión global sobre la causalidad de los daños patológicos. Es el caso de Johnson (17), Addleson (18), Eldridge (19), PSA (20), Monjo (21) y Díaz, Lloverá y Noró (22), entre otros, aunque uno de nosotros ya presentó un avance al respecto (16).

En nuestro modelo, separamos causas de orígenes y defectos de errores. En las primeras, distinguimos las acciones que causan el fallo, o el daño, y las disposiciones constructivas sobre las que éstas actúan. Estas disposiciones darán una respuesta prestacionalmente insuficiente si las acciones son excesivas, las disposiciones defectuosas, o si coinciden ambas circunstancias. Las causas de los fallos, y de los daños, son dichos excesos o defectos. Para valorar si una solución constructiva es defectuosa, o no, necesitamos un patrón de referencia con el que compararla. Tenemos que probar que la disposición constructiva real se separa significativamente del patrón de referencia, y que esta diferencia causa los daños, porque no toda diferencia con el patrón es patológicamente relevante, ni aunque lo fuera tiene por qué ser causa de los daños observados.

Los defectos constructivos pueden tener su origen en defectos de promoción, de proyecto, de la dirección de la obra, de la dirección de la ejecución, del suministro de materiales, de la ejecución y de la conservación y utilización, según los casos y sin que sean incompatibles unos con otros. A este respecto, la LOE defiende la individualización de responsabilidades, salvo en casos específicos que permite la responsabilidad solidaria. Podría pensarse que la responsabilidad guarda relación directa con la naturaleza de los daños: que el arquitecto tendría que responder de los que afectasen a la estructura; el arquitecto técnico de los que afectasen a la habitabilidad, y el constructor de los que afectasen a elementos de terminación o acabado. Esto no es cierto. La LOE distingue plazos de garantía en función de los requisitos afectados, pero no establece el agente individualmente responsable de éstos. En cualquier caso, asignar responsabilidades es labor jurídica, nunca técnica, que solo debería referirse al origen del daño en función del resultado de las intervenciones profesionales diferenciadas de los agentes de la edificación, pero no directamente referidas a uno o más agentes particulares. El técnico interpretará los documentos técnicos para determinar en

\footnotetext{
${ }^{11}$ La argumentación sobre el fallo contradice la del daño, intencionalmente, para mostrar un ejemplo de argumentación insuficiente (la del daño).

12 Indicamos con corchetes los números que se encuentran en los vértices de la representación gráfica planteada.

13 Jiménez Montoya, P., García Meseguer, A., Morán Cabré, F., Arroyo Portero, J.C. (2010), Hormigón armado, Barcelona, Gustavo Gili (ed.15).
} 
qué fase de la obra se decidió que algo se construyera o se usara como se ha hecho para originar el daño, pero no quién lo hizo. El médico forense explica cómo ha muerto la víctima, no quién es el asesino.

\section{Causa}

- $\mathbf{P}_{\mathbf{c}}$ : La forma del vierteaguas [1] es la causa de las filtraciones de lluvia en el antepecho de la ventana [2].

- $\mathbf{S}_{\mathbf{c}}$ : El vierteaguas [1] apenas vuela $1 \mathrm{~cm}$ del paramento exterior, carece de goterón y de pendiente [3].

- $\mathbf{G}_{\mathbf{c}}$ : Los parámetros técnicos del vierteaguas [3] incumplen las especificaciones del CTE DB-HS1 $\mathrm{a}^{0}$ 2.3.3.6 [4].

- $\mathbf{F}_{\mathbf{c}}$ : Implícito: El CTE DB-HS1 [4] es de obligado cumplimiento y su finalidad es, entre otras, garantizar la protección del edificio frente a las filtraciones de lluvia [2].

\section{Origen}

- $\mathbf{P}_{\mathbf{o}}$ : La forma del vierteaguas [1] se genera en un defecto de proyecto [2].

- $\mathbf{S}_{\mathbf{o}}$ : El vierteaguas [1] consiste en una caja de chapa, sin pendiente ni goterón, pero con $4 \mathrm{~cm}$ de vuelo [3].

- $\mathbf{G}_{\mathbf{o}}$ : Las características técnicas descritas [3] coinciden con las especificadas en el proyecto de ejecución [4].

- $\mathbf{F}_{\mathbf{o}}$ : El proyecto de ejecución [4] es el conjunto de documentos en los que el proyectista justifica técnicamente que las soluciones propuestas deberían carecer de defectos [2].

La representación gráfica de estos dos argumentos sigue la misma forma que la Figura 2.

\subsection{Argumentar sobre la reparación y su coste}

En patología en edificación las mayores discrepancias entre técnicos suelen aparecer en las propuestas de reparación y, sobre todo, en sus mediciones y valoraciones económicas, pues con frecuencia su justificación es insuficiente o subjetiva.

El proponer una reparación, el técnico debería tener muy presentes estos dos criterios:

1. Coherencia._La reparación tiene que corresponder al diagnóstico y al pronóstico. El técnico indicará las actuaciones de emergencia para estabilizar la progresión de los daños, si se requieren, las de subsanación o eliminación de la causa, y las de reparación de los daños.

2. Concepto. El técnico deberá asegurarse de que la reparación que propone, en concepto y extensión, elimina la causa y repara los daños realmente. Entran en juego en este paso los conocimientos técnicos del perito, las referencias a la bibliografía y a la normativa técnica existente, las experiencias previas, etc.

Para ser trasparente en su valoración, el técnico desglosará los conceptos incluidos; por ejemplo, si valora Seguridad y Salud, o si calcula ejecución material o por contrata, explicitará el IVA, los honorarios profesionales si son necesarios, impuestos municipales, etc.

\section{Reparación $^{14}$}

- $\mathbf{P}_{\mathbf{r}}$ : Rehacer la impermeabilización de las juntas de dilatación [1] eliminará las humedades del garaje [2].

- $\mathbf{S}_{\mathbf{r}}$ : Esta intervención [1] implica demoler el pavimento para descubrir la junta, rehacer su impermeabilización, y volver a pavimentar [3].

- $\mathbf{G}_{\mathbf{r}}$ : La reparación descrita [3] se hará conforme a las especificaciones del CTE DB-HS1, $\mathrm{a}^{0}$ 2.4.4.1.1 [4].

- $\mathbf{F}_{\mathbf{r}}$ : Implícito: Como en el ejemplo de la causa $\left(\mathrm{F}_{\mathrm{C}}\right)$.

La representación gráfica de esta argumentación sigue la misma forma que la figura 2.

\section{Valoración económica ${ }^{15}$}

- $\mathbf{P}_{\mathbf{v}}$ : La reparación propuesta [1] se valora en 10.716,76€ $€^{16}$ [2].

- $\mathbf{S}_{\mathbf{v}}$ : La reparación propuesta [1] se descompone en conceptos y partidas de obra con mediciones desglosadas en partes de obra identificadas [3A], a las que corresponden precios unitarios descompuestos [3B].

- $\mathbf{G}_{\mathbf{v}} \mathbf{1}$ : Las mediciones [3A] se han obtenido sobre el edificio y sus planos, que se adjuntan marcando las zonas afectadas [4A].

- $\mathbf{G}_{\mathbf{v}}$ 2: Los precios unitarios [3B] se han obtenido a partir de precios descompuestos adjuntos y de la base Precio Centro [4B].

- $\mathbf{F}_{\mathbf{v}} \mathbf{1}$ y $\mathbf{F}_{\mathrm{v}}$ 2: No se requiere: La objetividad del procedimiento de referencia [4A-4B] para obtener el valor de la reparación [2] es generalmente aceptada en el contexto en el que se elabora el dictamen.

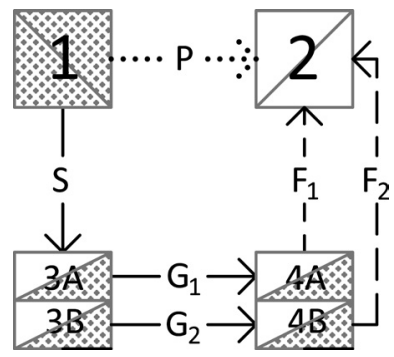

Figura 5. Representación del argumento de la valoración económica de la subsanación de la causa.

La figura 5 muestra cómo la garantía está formada por dos argumentos en paralelo, relativos a las mediciones y a los precios, que no son complementarios, sino conjuntos o simultáneos.

Hemos indicado los esquemas argumentales para los temas esenciales de un dictamen de patología pensando en su redacción ex novo, pero además este modelo y sus representaciones gráficas sirven para contrarrestar argumentaciones contrarias, aunque esto ya queda fuera de los objetivos de esta publicación.

\section{CONCLUSIONES}

El modelo de Toulmin se puede aplicar a la argumentación técnica en patología de la edificación manteniendo sus elementos principales, pero flexibilizando su rigidez formal.

\footnotetext{
${ }^{14}$ Nos referimos en este ejemplo a la subsanación de la causa, exclusivamente.

15 El lector puede suponer los detalles de las mediciones, planos y precios.

16 Se indica una cantidad cualquiera a modo de ejemplo.
} 
Podemos tratar estos elementos como pasos argumentales de cada uno de los temas principales del dictamen técnico en este campo, y disponer de un modelo coherente, completo y lógico para la argumentación técnica. Tales temas son $\left(1^{\circ}\right)$ demostrar o negar la existencia de daños materiales y sus fallos prestacionales asociados, $\left(2^{\circ}\right)$ explicar su causa y origen, y $\left(3^{\circ}\right)$ proponer una reparación indicando su coste. Aplicando el esquema de Toulmin, la argumentación técnica relativa a cada uno de los temas anteriores consta, en esencia, de una pretensión, que califica o predica un valor de cada uno de los objetos anteriores, apoyada por el soporte proporcionado por los datos o parámetros técnicos descriptivos del objeto, cuya pertinencia y valoración técnica se garantiza por comparación con un patrón objetivo que, a su vez, sirve para fundamentar la pretensión mediante su referencia genérica al predicado o finalidad de ésta, cerrando así la argumentación.

Así, basándonos en el modelo de Toulmin, podemos establecer un argumento cerrado en el que lo que pretendemos demostrar queda justificado o probado en los campos físico o material (soporte), técnico (garantía), y científico y jurídico (fundamento), que son a los que se debe ajustar todo dictamen técnico.

Para facilitar el análisis de argumentaciones contrarias, y detectar mejor sus carencias y errores lógicos, hemos mostrado una representación gráfica conceptual y sencilla del contenido y desarrollo de una argumentación, en la que representamos los cuatro conceptos principales, objeto [1], calificación pretendida [2], datos [3] y patrón de referencia [4], así como los argumentos elementales que los ligan: pretensión $(1 \rightarrow 2)$, soporte $(1 \rightarrow 3)$, garantía $(3 \rightarrow 4)$ y fundamento $(4 \rightarrow 2)$. Con esta herramienta gráfica es posible, además, representar argumentaciones complejas que utilicen argumentos encadenados, o en serie, en los que deben cumplirse varias condiciones simultáneamente para que el argumento quede cerrado, así como argumentos complementarios o en paralelo, en los que un argumento queda formado por otros complementarios o conjuntos.
Finalmente, recordamos que la ciudadanía exige una cultura de transparencia creciente en todos los ámbitos. Especialmente en el caso de los procesos judiciales, la mediatización obliga a potenciar dicha transparencia. Es necesario avanzar en la utilización de técnicas y herramientas expositivas, críticas y de análisis, que favorezcan una argumentación más transparente en la que la credibilidad del autor no se base exclusivamente en su autoridad.

En origen, el esquema de argumentación basado en la metodología de Toulmin no está dirigido al campo de los dictámenes relativos a patología de la edificación, pero sirve para poder identificar los puntos fuertes y débiles de las argumentaciones en este campo. Aunque todo análisis argumentativo implica cierta interpretación personal, lo importante de un método es si a través de él se consigue analizar críticamente y con claridad las argumentaciones propias y ajenas y, en consecuencia, es más fácilmente entendido por el destinatario del dictamen.

El modelo propuesto trata de aportar una solución al problema de saber si una afirmación técnica ha quedado plenamente argumentada, o no. En general, la investigación aquí presentada nos dota de herramientas con las que desarrollar la argumentación técnica en los dictámenes periciales relativos a patología en edificación. Como tal, nos permite ordenar la mente y, en este sentido, cumple su función, porque el primer paso para ser claro es verlo claro; aun así, queda mucho por delante en el campo de la argumentación técnica.

Hemos mostrado varios ejemplos sencillos de la aplicación de este modelo de argumentación y de su representación gráfica a cada uno de los temas principales del dictamen de patología de la edificación, modelo que hemos utilizado comprobando que es una poderosa y útil herramienta que ayuda tanto a la hora de elaborar una argumentación técnica propia como a la de discutir otra ajena. Argumentar según el modelo que proponemos no nos garantiza hacerlo bien, pero nos ayuda a no hacerlo mal.

\section{REFERENCIAS}

(1) Lasheras Merino, F., Gómez-Martinho Palacio, L. (2017, 10 de marzo). El espacio de la argumentación técnica en el dictamen pericial de patología de la edificación. Sus planos dialéctico, lógico y retórico. En CITE 2017, Madrid.

(2) García Meseguer, Á. (1963). Léxico de la construcción. Informes de la Construcción, 16(150): 53-58. https://doi. org/10.3989/ic.1963.v16.i150.4715

(3) García Meseguer, Á. (1985). La patología y el lenguaje. Informes de patología. Informes de la Construcción, 37(376): 5-16. https://doi.org/10.3989/ic.1985.v37.i376.1799

(4) Calavera Ruiz, J. (2009). Manual para la redacción de informes técnicos en construcción, Madrid: INTEMAC.

(5) Guerra San Martín, J. (1984). El perito arquitecto en el ordenamiento procesal español: regulación y sugerencias. Informes de la Construcción, 34(357): 47-56. https://doi.org/10.3989/ic.1984.v35.i357.1941

(6) Marín García, D. (2005). Modelo gráfico para la peritación judicial de patologías en edificación (Tesis doctoral). Universidad de Sevilla.

(7) León Cascante, I. (2014). Análisis de casuística de peritaciones arquitectónicas en procesos judiciales (Tesis doctoral). Universidad del País Vasco.

(8) Matos de Araujo Chaves, M. (2012). El dictamen pericial: criterios de valoración y su motivación en la sentencia civil (Tesis doctoral). Universidad de Burgos.

(9) Vázquez Rojas, C. (2014). La prueba pericial. Entre la deferencia y la educación (Tesis doctoral). Universidad de Gerona.

(10) Toulmin, S. (1958). The uses of argument, Cambridge: Cambridge University Press.

(11) Toulmin, S., Rieke, R., Janik, A. (1979). An introduction to reasoning. New York: Macmillan Publishing Co.

(12) Atienza, M. (2013). Curso de argumentación jurídica. Madrid: Trotta.

(13) García Meseguer, A. (1989). Control de calidad en construcción. Madrid: A.N.C.O.P.

(14) España. Cortes Generales (1999). Ley 38/1999 de Ordenación de la Edificación. Boletín Oficial del Estado, nº 266. 
(15) Real Decreto 314/2006 que aprueba el Código Técnico de la Edificación. Boletín Oficial del Estado, nº 74. España, modificado por RD 1371/2007 que aprueba el DB-HR Protección frente al ruido. Boletín Oficial del Estado, nº 254. España y RD 173/2010 que modifica el CTE en materia de accesibilidad y no discriminación de las personas con discapacidad. Boletín Oficial del Estado, $\mathrm{n}^{0}$ 61. España.

(16) Lasheras Merino, F. (2012, 12-14 abril). Sobre el concepto de causa en patología de la construcción, $4^{\circ}$ Congreso de Patología y Rehabilitación de Edificios, PATORREB. Santiago de Compostela: Colexio Oficial de Arquitectos de Galicia.

(17) Johnson, S.M. (1965). Deterioration, maintenance and repair of structures, Universidad de California: McGraw-Hill (vers. esp. 1973, Barcelona: ed. Blume).

(18) Addleson, L. (1978). AJ guide to building failures. Architectural Journal (vers. esp. 1986, Madrid, Hermann Blume, p.14)

(19) Eldridge, H.J. (1982). Common defects in buildings, London: H.M.S.O (vers. esp. 1982, Barcelona, Gustavo Gili).

(20) Property Services Agency (1989). Defects in buildings. London: H.M.S.O.

(21) Monjo Carrió, J. (1999). La patología y los estudios patológicos. En Curso de patología, conservación y restauración de edificios, Tomo 1 (pp. 11-41). Madrid: Colegio Oficial de Arquitectos de Madrid.

(22) Díaz C., Llovera S., Noró M. (2004). Dicionari de patología i manteniment d'edificis. Barcelona: Universitat Politècnica de Catalunya. 